\title{
Measurement of the Angle Between the Superior Mesenteric Artery and the Aorta: Correlation with Body Mass Index
}

\author{
Raza Sayani ${ }^{1}$, Mustafa Belal Hafeez Chaudhry ${ }^{1}$, Mohammad Hasan ${ }^{2}$, Ali Janjua ${ }^{3}$, Irfan Nazir ${ }^{3}$, \\ Kumail Khandwala ${ }^{1, *}$ \\ ${ }^{1}$ Department of Radiology, Aga Khan University Hospital, Karachi, Pakistan \\ ${ }^{2}$ Department of Medicine, Jinnah Sindh Medical University, Karachi, Pakistan \\ ${ }^{3}$ Medical College, Aga Khan University, Karachi, Pakistan
}

Email address:

kumail.khandwala@gmail.com (K. Khandwala)

${ }^{*}$ Corresponding author

\section{To cite this article:}

Raza Sayani, Mustafa Belal Hafeez Chaudhry, Mohammad Hasan, Ali Janjua, Irfan Nazir, Kumail Khandwala. Measurement of the Angle Between the Superior Mesenteric Artery and the Aorta: Correlation with Body Mass Index. International Journal of Medical Imaging. Vol. 9, No. 2, 2021, pp. 104-108. doi: 10.11648/j.ijmi.20210902.12

Received: February 24, 2021; Accepted: May 11, 2021; Published: May 20, 2021

\begin{abstract}
Objective: To discover the mean figures of the distance and angle between the superior mesenteric artery (SMA) and the aorta in four standard acknowledged body mass index (BMI) classifications in an ordinary populace and for the two genders, to work with a computed tomography CT based finding of Superior mesenteric artery syndrome (SMAS). Methods: A retrospective cross-sectional study was performed on 338 (157 female, 181 male) patients who were referred for abdominal CT examination. Age, sex, weight and height of all patients were noted, and the BMI was calculated. The distance between the SMA and aorta were measured, as was the angle. Pearson and Spearman correlation coefficients were used to correlate distance and BMI category, angle and BMI category, anteroposterior abdominal distance and SMA angle with aortomesenteric distance. Results: The mean BMI of patients in our study was $27.95 \mathrm{~kg} / \mathrm{m}^{2}$ while the mean SMA angle, mean anteroposterior abdominal wall distance and mean aortomesenteric distance was 58.74 degrees, $224.35 \mathrm{~cm}$, and 18.98 $\mathrm{cm}$, respectively. The results showed positive correlation of BMI and anteroposterior abdominal distance with SMA angle and distance. This means if BMI increases, SMA angle and aortomesenteric distance will also increase, and vice versa. This same relationship was also valid for anteroposterior abdominal distance with aortomesenteric angle and distance. Conclusion: Our study helps in stating the values of aortomesenteric distance and angle for different BMI categories that can be used in CT-based diagnosis of SMAS in a South Asian population. The values we found for different BMI categories can be used by radiologists as a reference standard.
\end{abstract}

Keywords: Body Mass, Superior Mesenteric Artery Syndrome, BMI, Computed Tomography, Aorta, Angle, Distance

\section{Introduction}

Superior mesenteric artery syndrome (SMAS) is an uncommon reason for upper gastrointestinal obstruction where the third bit of the duodenum is caught between the aorta and the superior mesenteric artery (SMA). Patients experiencing the condition regularly present with concerning weight reduction and epigastric agony because of hindrance of the third piece of duodenum [1]. Cardiac cachexia is one of its significant complexities which is for the most part excluded by numerous caretakers [2].

There has been a positive relationship with pressure of the third piece of the duodenum by the SMA with anatomical or mechanical elements and to intense or ongoing decrease of the retroperitoneal fat [3]. Related mechanical components are for the most part significant weight reduction, for example, anorexia nervosa, malabsorption, or hyper-catabolic states like burns, invasive procedures, critical wounds, or malignancies [2]. The intense angulation of the SMA 
secondary to the deficiency of retroperitoneal fat cushioning brings about choking at the area where the duodenum crosses and accordingly triggers the condition [3].

There are a couple of focuses in literature that we need to consider: the first is that there is a significance of the distance and angle of the SMA from the aorta in the etiology of SMAS [2-9]; and furthermore retroperitoneal fat effects the angle between the SMA and aorta itself $[3-5,8]$. Body mass index (BMI) itself is a gadget for assessing underweight and overweight individuals and offers a nice hint of muscle to fat proportion. Thusly, in this study we meant to discover the mean upsides of the distance and angle between the SMA and aorta in four standard acknowledged BMI classes, in an ordinary populace and in the two genders, to work with a computed tomography (CT) based determination of SMAS.

\section{Materials \& Methods}

We performed a retrospective, cross-sectional study in which we collected the relevant data of 338 patients who were referred to our department for abdominal CT examinations due to different reasons between 2013 and 2018. The major inclusion criteria were any patient admitted for abdominal CT scan at the Aga Khan University at the department of radiology, and the exclusion criteria included patients in acute state of post-surgery. We intended to keep the female to male ratio $1: 1$. Informed consent prior to the start of procedure was taken from each patient so that their data could be used in future for research purpose. The age, sex, weight and height of all patients was noted, and the BMI was calculated as the weight $(\mathrm{kg})$ divided by the square of the height [weight $(\mathrm{kg}) /$ height $\left(\mathrm{m}^{2}\right)$ ]. We divided the BMI into four categories as accepted by the World Health Organization (WHO) as follows [10]: Category I: BMI $<18.5$.

Category II: BMI 18.5- 24.9

Category III: BMI 25-29.9

Category IV: $\mathrm{BMI}>30$

\subsection{CT Technique and Measurements}

Multi-cut helical CT was performed on a 16-cut CT scanner (Somatom Sensation 16, Siemens, AG, Erlanger, Germany). Gantry turn time was 0.5 seconds. A tube voltage of $140 \mathrm{kVp}$ and a tube current in the scope of 100 and 380 $\mathrm{mA}$ were utilized in all patients, contingent upon the size of the patient. All patients at first got $800-1,000 \mathrm{~mL}$ of diatrizoate meglumine (Urovist-AngiograWn; Schering, Germany) weakened to a $2-3 \%$ concentration and given orally around a 45-minute stretch. Standard intravenous contrast media protocol included $2 \mathrm{mg} / \mathrm{kg}$ of iopromide (Ultravist 300, Schering AG. Berlin, Germany) infused by a mechanized injector (CT Injector; Ulrich Medical, UlmJungingen, Germany) at a pace of $2 \mathrm{~mL} / \mathrm{s}$ or by hand infusion. As per our standard stomach CT protocol, $10 \mathrm{~mm}$ sections were gained with addition of $8 \mathrm{~mm}$, and the patients were set in the supine position. Resulting reproduced essential pictures of $2 \mathrm{~mm}$ cut thickness were gained using a medium-sharp convolution piece $(\mathrm{B} 30 \mathrm{f})$ with an image organization of 512 x 512 pixels. All the image data were sent electronically to a workstation (Leonardo, Siemens AG, Erlanger, Germany) for analysis. From these pictures sagittal or angled sagittal multiplanar reconstruction (MPR) pictures were acquired for evaluation of the expanding design of the SMA from the aorta.

The angles and the distances between the SMA and the aorta were estimated by two clinical undergraduates and the outcomes were noted in an agreement style for statistical investigation. The distance between the SMA and aorta was estimated as the extreme distance between the foremost edge of the aorta and the posterior aspect of the SMA at a level where the duodenum visually crossed, on axial sections. The angle between those vessels was calculated on reformatted sagittal-angled sagittal pictures at a similar level. For point estimations, a line was drawn between the foundation of SMA and an imaginary point on the SMA where the SMA starts to drop parallel to the abdominal aorta. Calculations were acquired by electronic calipers. The angles were gotten by manual tracing and the degrees were electronically determined.

\subsection{Statistical Analysis}

For every gender, mean upsides of distance and angle estimations were determined with standard deviations and $95 \%$ confidence intervals. For every gender, Pearson connection coefficients were determined between the aortomesenteric distance and BMI, SMA angle and BMI, anteroposterior abdominal wall distance and SMA angle as well as anteroposterior abdominal wall distance and aortomesenteric distance. Spearman correlation coefficients were also calculated between the aortomesenteric distance and BMI category and SMA angle and BMI category [10]. Correlation coefficients $0.1<\mathrm{r}<0.3,0.3<\mathrm{r}<0.5$ and $>0.5$ were used as an indication of weak or low, moderate and strong associations, respectively [11].

\section{Results}

An aggregate of 338 patients were analysed for our study who presented with different complains among which staging of malignancy (30\%) was the commonest, followed by other complaints, mainly abdominal pain, surveillance/followup/re-staging of some malignancy, and abdominal infection (e.g., tuberculosis, Whipple's disease). Only a single patient was diagnosed with SMAS with an SMA angle of 13 degrees. Gender division was 157 females and 181 males resulting in almost 1:1 female: male ratio. Majority of our patients were in the age group of 51-60 years. They fell mainly in category IV of BMI (i.e., $>30 \mathrm{~kg} / \mathrm{m}^{2}$ ), which was shared by $35.5 \%(n=120)$ of our patients (Table 1$)$. Overall, mean BMI of patients in our study was $27.95 \mathrm{~kg} / \mathrm{m}^{2}$ while the mean SMA angle, mean anteroposterior abdominal wall distance and mean aortomesenteric distance was $58.74 \mathrm{~cm}$, $224.35 \mathrm{~cm}$, and $18.98 \mathrm{~cm}$, respectively. 
Table 1. Number of patients and mean value ( \pm standard deviation) of their ages among different BMI categories.

\begin{tabular}{lllll}
\hline \multirow{2}{*}{ BMI category } & Male & & & \\
\cline { 2 - 5 } & I & II & III & IV \\
\hline $\mathrm{N}$ (total) & 14 & 68 & 54 & 45 \\
Mean age (years) & $44.93 \pm 25.65(20-77)$ & $56.19 \pm 15.94(7-83)$ & $53.57 \pm 15.48(17-82)$ & $53.56 \pm 14.45(22-87)$ \\
\hline
\end{tabular}

Table 1. Continued.

\begin{tabular}{|c|c|c|c|c|}
\hline \multirow{2}{*}{ BMI category } & \multicolumn{4}{|l|}{ Female } \\
\hline & I & II & III & IV \\
\hline $\mathrm{N}$ (total) & 3 & 40 & 39 & 75 \\
\hline Mean age (years) & $46.67 \pm 18.23(27-63)$ & $53.12 \pm 14.60(24-80)$ & $51.95 \pm 13.55(23-78)$ & $54.19 \pm 11.20(26-78)$ \\
\hline
\end{tabular}

BMI: body mass index

$95 \%$ confidence intervals were used to evaluate the mean values of aortomesenteric distance, angle and anteroposterior abdominal wall distance which are shown in Table 2 (females) and Table 3 (males).

Table 2. Mean values ( \pm standard deviation) of aortomesenteric distance, SMA angle, and anteroposterior abdominal wall distance measurements in different BMI categories of female patients.

\begin{tabular}{lllll}
\hline & BMI & & & IV \\
\cline { 2 - 5 } & I & II & III & $15.14 \pm 4.38(8-27)$ \\
\hline Aortomesenteric distance $(\mathrm{cm})$ & $13.30 \pm 5.40(9-19)$ & $14.51 \pm 5.25(6-32)$ & $19.24 \pm 6.69(5-38)$ \\
SMA angle (degrees) & $48.50 \pm 15.65(32-63)$ & $46.48 \pm 20.00(20-109)$ & $54.71 \pm 43.06(20-296)$ & $55.13 \pm 20.17(13-128)$ \\
Anteroposterior abdominal distance $(\mathrm{cm})$ & $195.5 \pm 72.5(153-279)$ & $189.6 \pm 34.3(43-250)$ & $224.2 \pm 25.8(180-287)$ & $261.3 \pm 32.3(174-326)$ \\
\hline
\end{tabular}

BMI: body mass index

SMA: superior mesenteric artery

Table 3. Mean values ( \pm standard deviation) of aortomesenteric distance, SMA angle, and anteroposterior abdominal wall distance measurements in different BMI categories of male patients.

\begin{tabular}{lllll}
\hline & BMI & & & \\
\cline { 2 - 5 } & I & II & III & IV \\
\hline Aortomesenteric distance $(\mathrm{cm})$ & $12.86 \pm 6.05(7-27)$ & $17.96 \pm 5.88(6-33)$ & $22.14 \pm 8.29(11-59)$ & $25.90 \pm 7.23(12-48)$ \\
SMA angle (degrees) & $46.96 \pm 22.7(13-88)$ & $58.36 \pm 19.53(18-103)$ & $68.28 \pm 18.40(27-113)$ & $72.61 \pm 19.90(29-116)$ \\
Anteroposterior abdominal distance $(\mathrm{cm})$ & $151.4 \pm 15.0(118-169)$ & $196.3 \pm 37.04(23-276)$ & $223.8 \pm 25.9(160-305)$ & $261.46 \pm 31.00(182-321)$ \\
\hline
\end{tabular}

BMI: body mass index

SMA: superior mesenteric artery

For females, there was a weak, but significant and positive Pearson correlation between the SMA angle and BMI ( $\mathrm{r}-0.12$; $\mathrm{P}<0.001)$. Correlation between aortomesenteric distance and BMI was moderate and significantly positive ( $\mathrm{r}-0.34$; $\mathrm{P}<0.001)$. Correlation between anteroposterior abdominal wall distance and SMA angle was weak and significantly positive $(\mathrm{r}-0.11 ; \mathrm{P}<0.001)$. Correlation of anteroposterior abdominal wall distance with aortomesenteric distance was moderate and significantly positive $(\mathrm{r}-0.48 ; \mathrm{P}<0.001)$. Spearman correlation between the BMI category and SMA angle was weak, but significant and positive $(\rho-0.21$; $\mathrm{P}<0.001)$ and correlation between BMI category and aortomesenteric distance was moderate and significantly positive $(\rho-0.39 ; \mathrm{P}<0.001)$.

For males, Pearson correlation of BMI with SMA angle ( $\mathrm{r}-$ 0.36 ; $\mathrm{P}<0.001)$ or aortomesenteric distance $(\mathrm{r}-0.48 ; \mathrm{P}<0.001)$ was moderate and significantly positive. The correlation between anteroposterior abdominal distance and angle was weak, but significant and positive $(\mathrm{r}-0.29 ; \mathrm{P}<0.001)$. Correlation of anteroposterior abdominal distance with aortomesenteric distance was strong and significantly positive $(\mathrm{r}-0.55 ; \mathrm{P}<0.001)$. Spearman correlation between BMI category and SMA angle was moderate and significantly positive $(\rho-0.35 ; \quad \mathrm{P}<0.001)$ while with aortomesenteric distance the correlation was strong and significantly positive $(\rho-0.51 ; \mathrm{P}<0.001)$ (Figure 1$)$.

Overall, the results show positive correlation of BMI and anteroposterior abdominal distance with SMA angle and distance. This means if BMI increases, SMA angle and aortomesenteric distance will also increase, and vice versa. This same relationship is also valid for anteroposterior abdominal distance with aortomesenteric angle and distance.

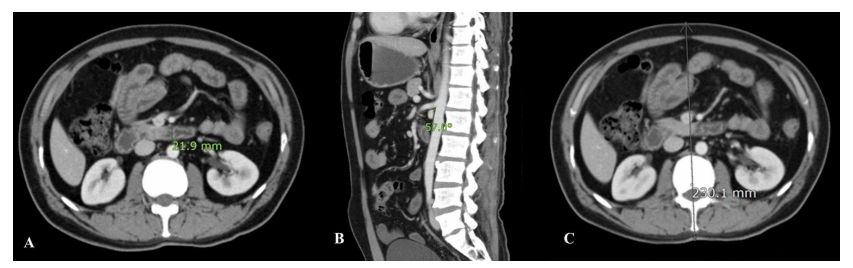

Figure 1. A) Axial CT image showing distance between superior mesenteric artery and aorta in a 39-year-old male with BMI of 28 (Category III). B) Sagittal CT image demonstrating superior mesenteric artery angle of 57 degrees and C) axial CT image showing the calculated anteroposterior abdominal wall distance in the same patient.

\section{Discussion}


SMAS (Wilkie's syndrome) is one of the rarest disorders of the abdomen that is usually overlooked by many physicians. Rokitansky was the pioneer in explicating this disorder in the mid-19th century [12]. In the late 20th century, the disorder was reported to be endemic in only those areas where professionals were well equipped with knowledge and procedural techniques that would help in the diagnosis and treatment of this entity [13]. Since then, a lot of work has been done in this regard.

Anatomy of the SMA is such that aorta gives off the SMA at the level of L1, which therefore makes it have an acute angle with the aorta. Position of this artery is in such a way that neck of pancreas lies in front of it, the superior mesenteric vein lies to the right of it, while uncinate process and third part of duodenum lie behind it [14]. SMA is crossed by left renal vein just below its origin. Decreased SMA angle not only compresses the duodenum, but at times compresses the left renal vein, resulting in nutcracker syndrome [14]. Normal range for the angle formed by the SMA is between 38 and $56^{\circ}$ and the mean radiographic aortomesenteric distance ranges from 10-28 $\mathrm{mm}$ [14]. A lot of literature is present regarding SMAS and its treatment; yet no study is present which states values of SMA angle and aortomesenteric distance for different BMI categories and anteroposterior abdominal wall distance. In this study, we aimed to investigate the correlation between BMI and anteroposterior abdominal wall distance with aortomesenteric angle and distance in our general South Asian population. Our major purpose was to set normal values of aortomesenteric distance and angle in our region, to set them as standard for CT-based diagnosis of SMAS.

There have been a variety of explanations for SMAS; Choi et al. [15] proposed that in addition to decreased SMA angle and aortomesenteric distance, the path followed by artery anterior and parallel to aorta is also a major factor contributing to SMAS. With variety of definitions of SMAS, its diagnosis and treatment are still challenging. In a study, Gustafsson et al. [16] found hypotonic duodenography with barium to be the most reliable and accurate method for diagnosing SMAS in patients with chronic upper abdominal symptoms, with discomfort being the only side effect. Neri et al. [9] emphasized the use of abdominal ultrasounds in patients with unexplainable and prolonged abdominal pain to diagnose SMAS. Lippl et al. [17] further showed the usefulness of ultrasounds in diagnosing SMAS with ease. Santer et al. and Applegate et al. then brought the usage of CT scans for the first time in diagnosing SMAS and demonstrated that dynamic thin-section CT with sagittal reconstruction is substantial in demonstrating SMAS $[4,18]$. Treatment and management of SMAS is contentious too as some believe supportive care (nutritional support, frequent small meals, prokinetics etc.) can drastically alleviate the symptoms, while others go for surgery [9, 19, 20].

According to us, major factors that make an individual susceptible to SMAS are the aortomesenteric angle and distance. Our viewpoint is supported by Unal et al. and Neri et al. who showed strong correlation between decreased aortomesenteric distance and angle, and clinical symptoms of SMAS [9, 21]. Ozkurt et al. further introduced BMI as an indicator in identifying individuals prone to suffer from SMAS [10]. The results of our study correspond with other south Asian studies in showing a strong correlation of BMI with aortomesenteric distance and angle [22]. We further added anteroposterior abdominal wall distance as an indicator which also showed a significant and positive correlation with aortomesenteric distance and angle. In this way, we can set mean values of SMA angle and distance in different BMI categories as a standard in our normal population to suspect SMAS.

One of the major limitations of our study was that it did not include many patients with confirmed diagnosis of SMAS which can else help us in describing the resemblance of aortomesenteric angle and distance with the normal ones. A large sample size could also play a vital role in standardizing the values. Additionally, general limitations with retrospective studies also apply. On the other hand, an almost equal number of males and females was one of the strengths of our study which helped to determine the differences in values as per gender.

\section{Conclusions}

Our study shows a positive correlation of BMI and anteroposterior abdominal distance with SMA angle and distance, and between anteroposterior abdominal distance and aortomesenteric angle and distance. Therefore, we suggest that these values obtained for aortomesenteric distance and angle for different BMI categories can be used in CT-based diagnosis of SMAS in a South Asian population and can be used by radiologists as a reference. However, further prospective and larger studies in this aspect could elevate the reliability.

\section{References}

[1] Merrett ND, Wilson R, Cosman P, Biankin AV: Superior mesenteric artery syndrome: diagnosis and treatment strategies. J Gastrointest Surg. 2009, 13: 287. 10.1007/s11605008-0695-4.

[2] Roy A, Gisel JJ, Roy V, Bouras EP: Superior mesenteric artery (Wilkie's) syndrome as a result of cardiac cachexia. J Gen Intern Med. 2005, 20: C3-C4. 10.1111/j.15251497.2005.0201.x.

[3] Matheus Cde O, Waisberg J, Zewer MH, Godoy AC: Syndrome of duodenal compression by the superior mesenteric artery following restorative proctocolectomy: a case report and review of literature. Sao Paulo Med J. 2005, 123: 151-3. 10.1590/S1516-31802005000300013.

[4] Applegate GR, Cohen AJ: Dynamic CT in superior mesenteric artery syndrome. J Comput Assist Tomogr. 1988, 12: 976-80. 10.1097/00004728-198811000-00013.

[5] Ahmed AR, Taylor I: Superior mesenteric artery syndrome.

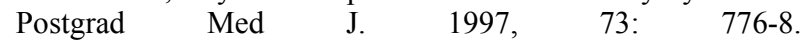
10.1136\%2Fpgmj.73.866.776. 
[6] Baltazar U, Dunn J, Floresguerra C, Schmidt L, Browder W: Superior mesenteric artery syndrome: an uncommon cause of intestinal obstruction. South Med J. 2000, 93: 606-8.

[7] Cohen LB, Field SP, Sachar DB: The superior mesenteric artery syndrome. The disease that isn't, or is it? J Clin Gastroenterol. 1985, 7: 113-6. 10.1097/00004836-19850400000002.

[8] Jani PG: Superior mesenteric artery syndrome: case report. East Afr Med J. 2003, 80: 334-6. 10.4314/eamj.v80i6.8712.

[9] Neri S, Signorelli SS, Mondati E, et al.: Ultrasound imaging in diagnosis of superior mesenteric artery syndrome. J Intern Med. 2005, 257: 346-51. 10.1111/j.1365-2796.2005.01456.x.

[10] Ozkurt H, Cenker MM, Bas N, Erturk SM, Basak M: Measurement of the distance and angle between the aorta and superior mesenteric artery: normal values in different BMI categories. Surg Radiol Anat. 2007, 29: 595-9. 10.1007/s00276-007-0238-9.

[11] Cohen J: Statistical power analysis for the behavioral sciences. Second (ed): Lawrence Erlbaum Associates, Hillsdale; 1988.

[12] Hines JR, Gore RM, Ballantyne GH: Superior mesenteric artery syndrome: diagnostic criteria and therapeutic approaches. Am J Surg. 1984, 148: 630-2. 10.1016/00029610(84)90339-8.

[13] Mansberger AR, Hearn JB, Byers RM, Fleisig N, Buxton RW: Vascular compression of the duodenum: emphasis on accurate diagnosis.. Am J Surg. 1968, 115: 89-96. 10.1016/00029610(68)90134-7.

[14] Welsch T, Büchler MW, Kienle P: Recalling superior mesenteric artery syndrome. Dig Surg. 2007, 24: 149-56. $10.1159 / 000102097$.
[15] Choi S, Pfalzer Jr F: Superior mesenteric artery syndrome. NY State J Med. 1976, 76: 986.

[16] Gustafsson L, Falk A, Lukes P, Gamklou R: Diagnosis and treatment of superior mesenteric artery syndrome. Br J Surg. 1984, 71: 499-501. 10.1002/bjs.1800710706.

[17] Lippl F, Hannig C, Weiß W, Allescher HD, Classen M, Kurjak M: Superior mesenteric artery syndrome: diagnosis and treatment from the gastroenterologist's view. J Gastroenterol. 2002, 37: 640-3. 10.1007/s005350200101.

[18] Santer R, Young C, Rossi T, Riddlesberger M: Computed tomography in superior mesenteric artery syndrome. Pediatr Radiol. 1991, 21: 154-5. 10.1007/BF02015638.

[19] Salem A, Al Ozaibi L, Nassif SMM, Osman RAGS, Al Abed NM, Badri FM. Superior mesenteric artery syndrome: A diagnosis to be kept in mind (Case report and literature review). Int J Surg Case Rep. 2017; 34: 84-86. 10.1016/j.ijscr.2017.03.018.

[20] Biswas A, Babu AA, Neelakantan S, Sarkar PS. Superior mesenteric artery syndrome: CT findings. BMJ Case Rep. 2016; 2016: bcr2016215885. Published 2016 Jun 28. $10.1136 / \mathrm{bcr}-2016-215885$.

[21] Ünal B, Aktas A, Kemal G, et al.: Superior mesenteric artery syndrome: CT and ultrasonography findings. Diagn Interv Radiol. 2005, 11: 90.

[22] Bhagirath Desai A, Sandeep Shah D, Jagat Bhatt C, Umesh Vaishnav K, Salvi B. Measurement of the distance and angle between the aorta and superior mesenteric artery on CT scan: values in Indian population in different BMI categories. Indian J Surg. 2015; 77 (Suppl 2): 614-617. 10.1007/s12262-0130941-1. 